\title{
Trombosis venosa esplácnica
}

\author{
Splanchnic venous thrombosis
}

\author{
Alexandre Pérez González, Julio Montes Santiago, Alberto Rivera Gallego \\ Servicio de Medicina Interna. Xerencia de Xestión Integrada de Vigo (EOXI. Vigo).
}

\section{Introducción}

La trombosis venosa esplácnica (TVE) es una expresión de la enfermedad tromboembólica venosa, que comprende la trombosis de las venas suprahepáticas (Síndrome de Budd-Chiari o $\mathrm{SBC}$ ), la trombosis venosa portal (TP), la trombosis de la vena mesentérica (TVM) y la trombosis de la vena esplénica (TVe).

La TVE es una entidad poco frecuente cuya incidencia estimada se sitúa en torno a los 5 casos por 10.000 habitantes, aunque es posible su infraestimación, dado que la detección incidental de la misma no es extraña. Las diversas formas clínicas de TVE presentan una fisiopatología, presentación clínica y pronóstico variable. La TP constituye la entidad más frecuente del grupo y se asocia en gran número de casos a la hepatopatía crónica. La TVe puede aparecer de manera aislada, especialmente en procesos infecciosos o inflamatorios del área hepatobiliar. La TVM en un importante número de casos se asocia a infarto intestinal, presentando una morbimortalidad elevada. Finalmente, el SBC se relaciona íntimamente con los procesos de hipercogulabilidad y los síndromes mieloproliferativos.

\section{Factores etiológicos}

Todos los componentes de la TVE tienen en común la aparición de una trombosis venosa a nivel abdominal, pero los factores de riesgo, tanto locales como sistémicos, difieren en gran medida (Tabla 1). Ocurre lo mismo con el pronóstico y con el tratamiento.

La TP es la entidad más frecuente. Su factor de riesgo mejor conocido es la cirrosis. Hasta un $20 \%$ de estos enfermos presentan en algún momento una TP. Las neoplasias abdominales, particularmente las derivadas del sistema hepatobiliar, constituyen otro factor de riesgo característico'.

En el SBC se identifica una causa subyacente hasta en el $80 \%$ de casos. En una gran parte se halla algún tipo de sindrome mieloproliferativo crónico (SMP)(policitemia vera, trombocitemia esencial). Este puede estar enmascarado por la presencia de esplenomegalia, que reduce los niveles séricos de hemoglobina y/o plaquetas, dificultando su diagnóstico. Entre un $26 \%$ y un $59 \%$ de pacientes estudiados se encuentran mutaciones en la tirosín-kinasa JAK2 (V617F), incluso en ausencia de SMP definido. Otras causas de SBC se relacionan con neoplasias que ejercen presión o invasión de las venas suprahepáticas (el más frecuente el hepatocarcinoma). Los anticonceptivos orales, el embarazo y los procesos inflamatorios abdominales constituyen otras causas frecuentes de SBC.
La TVe y TP aparecen conjuntamente frecuenemente, asociándose a neoplasias del área hepatobiliary a un mal pronóstico. La TVe aislada es menos común, pero puede darse en el seno de pancreatitis, tanto aguda como crónica, debido a factores inflamatorios locales. Hasta un $11 \%$ de los pacientes con pancreatitis crónica pueden desarrollar TVe. Algunos casos pueden presentar hipertensión portal y sangrado digestivo.

Dos son los procesos fundamentales en la TVM: inflamatorios (p. je. pancreatitis) e hipoperfusión. Las zonas más frecuentemente afectas son ileon y yeyuno, siendo poco común la afectación colónica o duodenal. La trombosis ocasiona un incremento retrógado de la presión venosa, flujo hacia el intersticio, edema tisular incrementando el riesgo de hemorragia, hypovolemia por secuestro y vasoespasmo (insuficiencia vascular e infarto intestinal).

Tabla 1. Agentes etiológicos de la Trombosis venosa esplácnica (TVE).

\begin{tabular}{|c|c|}
\hline $\begin{array}{l}\text { Estados de } \\
\text { hipercoagulabilidad }\end{array}$ & $\begin{array}{l}\text { Enfermedades } \\
\text { mieloproliferativas }\end{array}$ \\
\hline Mutación del factor V de Leiden & Policitemia vera \\
\hline $\begin{array}{l}\text { Mutación del gen de } \\
\text { la protrombina }\end{array}$ & Trombocitemia esencial \\
\hline Déficit de proteína C & Mutación de tirosín-kinasa JAK2 \\
\hline Déficit de proteína S & Mutaciones del gen CALR \\
\hline \multicolumn{2}{|l|}{$\begin{array}{l}\text { Hemoglobinuria } \\
\text { paroxística nocturna }\end{array}$} \\
\hline $\begin{array}{l}\text { Consumo de } \\
\text { anticonceptivos orales }\end{array}$ & Neoplasia \\
\hline \multirow[t]{5}{*}{ Embarazo } & Hepatocarcinoma \\
\hline & Neoplasias del área hepatobiliar \\
\hline & $\begin{array}{l}\text { Procesos inflamatorios } \\
\text { abdominales }\end{array}$ \\
\hline & Enfermedad inflamatoria intestinal \\
\hline & Pancreatitis aguda \\
\hline
\end{tabular}

\section{Estados de hipercoagulabilidad}

Múltiples causas de alteraciones de la coagulación, tanto congénitas como adquiridas, incrementan el riesgo de TVE. Existe una mayor relación con la SBE y en menor grado, con la TP. Los factores de riesgo más asociados al SBC son la mutación del factor $\mathrm{V}$ de Leiden, el déficit de proteína $\mathrm{C}$ y la mutación del gen de la protrombina. En la TP, el déficit más encontrado es la 
disminución de proteína $\mathrm{C}$, aunque también confieren riesgo la mutación del factor $\vee \mathrm{y}$ la del gen de la protrombina. El papel del déficit de proteína $\mathrm{S}$ es mucho más discreto.

\section{Síndromes mieloproliferativos crónicos (SMP)}

Los SMP son un conjunto de neoplasias hematológicas manifestadas por la hiperproducción de algún elemento de la serie mieloide. Muchos de estos procesos se asocian a SBC, y en menor grado TP. Los más frecuentes y mejor estudiados son la policitemia vera y la trombocitemia esencial. En estos casos merece especial atención la mutación V617F del gen JAK2. Esta alteración se encuentra presente en muchos pacientes afectos de SMP, sobre todo en la policitemia vera. Sin embargo, existe un apreciable número de pacientes que portan dicha mutación, pero no cumplen criterios definitorios de SMP, en los cuales se ha observado un incremento del riesgo, tanto de SBC, como de TP. También parece existir relación del SMP con mutaciones del gen CALR -que codifica la proteína calreticulina- ya que se detectan hasta en un $80 \%$ de los afectos de SMP con JAK2.

\section{Cirrosis hepática}

La cirrosis hepática es una patología frecuente en todo el mundo, aunque su causa difiere en relación con factores geográficos y socioeconómicos. Existen diferencias importantes en el papel de la cirrosis entre las diferentes subclases de TVE. En el caso de la TP, la cirrosis supone un importante factor de riesgo. La trombosis de la circulación portal ocasiona un incremento de la presión en este circuito, exacerbando el riesgo de sangrado digestivo, aumentando la cantidad de líquido ascítico y haciendo más probable la aparición de encefalopatía hepática. Además, se ha implicado como factor pronóstico, dificultando o impidiendo el transplante hepático ${ }^{29,30}$. En la TVe, cuando se asocia a TP, suele existir una cirrosis hepática subyacente.

La asociación cirrosis y SBC es infrecuente y, cuando se presenta cirrosis, parece más una consecuencia puesto que la trombosis de las venas suprahepáticas, puede ocasionarla a largo plazo ${ }^{6}$.

En el caso de la TVM la asociación es menos frecuente.

\section{Neoplasias}

Las neoplasias suponen una causa frecuente de TVE, sobre todo TP y SBC, y en menor medida, TVe y TVM22. Dos son Ios mecanismos fundamentalmente descritos: en primer lugar por compresión (sobre todo en la SBC) 0 infiltración/invasión (hepatocarcinoma en la $\mathrm{TP}^{13} 0$ neoplasias de vías biliares, pancreáticas 0 gástricas en la TP, TVe 0 TVM ${ }^{31}$ ) de los vasos venosos y, en segundo lugar, por alteración de la coagulación ${ }^{30}$.

\section{Procesos infecciosos e inflamatorios}

Dentro de este grupo se encuadran enfermedades de etiología infecciosa (p. ej. ej colecistitis) y procesos inflamatorios como la pancreatitis aguda y las enfermedades de Behçet ${ }^{33}$, inflamatoria intestinal y celíaca ${ }^{4,18,34}$. Todos presentan una vía fisipatológica común con un incremento de los factores proin- flamatorios y procoagulantes. Se relacionan en mayor grado con la TP, TVe y TVM y, en menor, con el SBC. En Asia la esquistosomiasis supone una causa no infrecuente de esta).

\section{Otros factores etiológicos}

Recientemente se ha observado que la prevalencia de hemoglobinuria paroxística nocturna (HPN) es elevada en pacientes con $\mathrm{SBC}^{23}$. En la HPN la activación del sistema del complemento induce hemólisis, en ocasiones masiva, incrementando el riesgo de trombosis venosa y arterial.

El embarazo y los anticonceptivos orales incrementan el riesgo de SBC, aunque no está claro que aumenten el riesgo de TP 0 TVM ${ }^{15}$. La infección por citomegalovirus es una causa rara de TVE.

\section{Tratamiento}

El tratamiento de la TVE es un tema controvertido. No existen ensayos clínicos aleatorizados acerca de la anticoagulación en estos pacientes. Su uso se basa en estudios observacionales, tanto retrospectivos como prospectivos que han proporcionados las bases de las recomendaciones recogidas en las Guías Clínicas (Figura 1). La eficacia del tratamiento anticoagulante, así como su seguridad, parece depender en enorme medida de la causa de la trombosis. La mayor parte de estudios se han centrado en SBC y TP no tumoral (cirróticos y no cirróticos).

\section{Síndrome de Budd Chiari}

La eficacia del tratamiento y, por lo tanto el pronóstico, van a depender de la identificación de la causa. Como se recalcó previamente, el SBC está íntimamente ligado a los SMP. AIgunos trabajos retrospectivos muestran que un inicio precoz de la anticoagulación y del tratamiento de la hemopatía condicionan un pronóstico más favorable ${ }^{23}$. La mayor parte de estos pacientes requerirán anticoagulación indefinida.

Los fármacos recomendados son la heparina de bajo peso molecular (HBPM), realizando transición hacia los fármacos con acción antivitamina K (AVK) a los 5-7 días del inicio del tratamiento. Existe sin embargo riesgo hemorrágico, especialmente en aquellos pacientes con hipertensión portal y varices esofágicas. Por ello, se aconseja un despistaje y tratamiento previo de estos procesos.

En caso de fracaso de la anticoagulación debe considerarse tratamiento endovascular, bien mediante inoculación local de trombolíticos, bien mediante angioplastia. La recanalización, aunque parcial, favorece un flujo adecuado y disminuye el riesgo de complicaciones a largo plazo.

En caso de fracaso o contraindicación de la angioplastia debe considerarse la derivación venosa 0 el trasplante hepático. En las últimas décadas ha emergido con fuerza la derivación portosistémica intrahepática transyugular (TIPS), que presenta menor tasa de complicaciones que las técnicas quirúrgicas y con buenos resultados a corto plazo.

\section{Trombosis venosa portal}

El objetivo del tratamiento en la TP es lograr la recanalización de la vena porta. Los estudios indican que un inicio 
Figura 1. Algoritmo propuesto para la anticoagulación en la TVE. Elaboracion propia a partir de la ref.36 (con permiso).

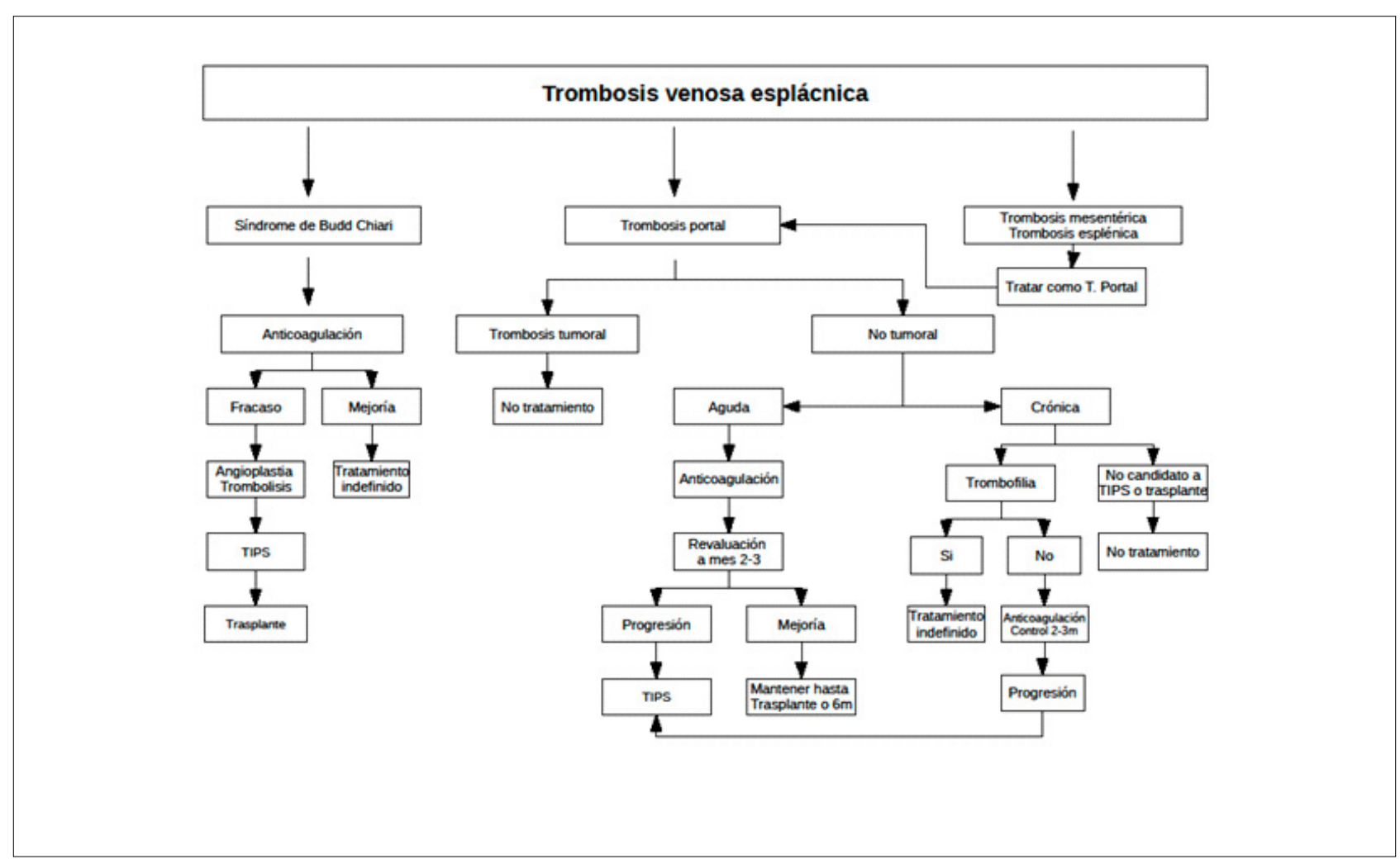

precoz de la misma podría favorecer un mejor pronóstico y tasa de recanalización.

La gran mayoría de los trabajos se han centrado en la TP no tumoral, en pacientes cirróticos y no cirróticos. En primer lugar, a la hora de instaurar o no un tratamiento debe considerarse si la trombosis es aguda o crónica. En caso de la trombosis aguda no tumoral, parece razonable instaurar tratamiento anticoagulante, con el objetivo de repermeabilizar la porta. Los fármacos más utilizados inicialmente son HBPM. En nuestro centro la tasa de recanalización de la TP no tumoral se situó en torno al $60 \%$ (datos no publicados). Tras un periodo de 5-7 días, una parte de pacientes realizaron transición a AVK. El tratamiento se mantendría un total de 2-3 meses, tras lo cual es aconsejable realizar un estudio de imagen de control. En caso de progresión de la trombosis, debe considerarse terapia de derivación (TIPS) ${ }^{42}$. Si el paciente no es candidato a estas técnicas y no se ha producido mejoría de trombosis, parece razonable suspender el tratamiento anticoagulante. Si a los 2-3 meses hay mejoría, debe considerarse prolongar tratamiento anticoagulante, incluso indefinido. Como se ha comentado, es importante realizar el diagnóstico y tratamiento adecuado de las varices esofágicas o gástricas para minimizar los riesgos de complicaciones ${ }^{37}$.

Si existen perspectivas de trasplante hepático, puede mantenerse el tratamiento hasta la cirugía, para estabilizar o mejorar en lo posible el área de trombosis.

En el caso de trombosis crónica, dependerá de las opciones terapéuticas. Si no es candidato a trasplante, no se aconseja el tratamiento anticoagulante. En caso de expectativa de trasplante se aconseja anticoagulación por los motivos previamente expuestos, pudiendo realizarse con HBPM o AVK. Apenas existen estudios acerca de la anticoagulación en la trombosis portal tumoral, así como del riesgo hemorrágico, pero la decisión probablemente dependa del pronóstico atribuido a la neoplasia.

En nuestra experiencia, en la TP tumoral la supervivencia a 6 meses fue $<10 \%$ (datos no publicados).

\section{Trombosis venosa mesentérica}

En líneas generales, en la TVM se aconseja anticoagulación precoz durante unos 2-3 meses, a fin de repermeabilizar el vaso. No existe evidencia de anticoagulación en la trombosis tumoral.

\section{Trombosis venosa esplénica}

En el caso de la TVe asociada a TP, se siguen los mismos consejos que en esta última. En el caso de la TVe aislada hay que examinar la causa subyacente. En los cuadros inflamatorios o infecciosos desencadenantes, se debe iniciar anticoagulación, aunque no está claro el plazo exacto. Los estudios varían en tratamientos de 306 meses. En el caso de SMP o alteraciones de la coagulación ha de ser valorado tratamiento prolongado o incluso indefinido, sin que existan ensayos clínicos al respecto ${ }^{37}$.

En la TVe tumoral existe la misma incertidumbre que en la TP o la TVM. No hay recomendaciones específicas al respecto, pero parece razonable que la instauración de tratamiento anticoagulante dependa del pronóstico de la neoplasia. 


\section{Conclusiones}

La TVE es una entidad hasta ahora considerada poco frecuente, pero cuya incidencia parece incrementarse en las últimas décadas, probablemente con la realización de estudios radiológicos altamente sensibles. Comprende un grupo heterogéneo de trombosis, con diferencias causales, clínicas y pronósticas notables.

Ante el diagnóstico de SBC se debe buscar una causa subyacente, especialmente SMP (estudio JAK2) V617F o trastornos de la coagulación. Aunque también HPN y consumo de anticonceptivos u otros productos hormonales.

En el caso de la TP, su relación con la cirrosis está bien definida, pero su tratamiento no está bien establecido. Las Guías Clínicas, basadas en estudios observacionales, aconsejan por lo general anticoagulación. Se propone también anticoagulación cuando la causa de la TP es resoluble 0 controlable (pancreatitis, infecciones, etc). Más incertidumbre aún subsiste en la TP tumoral. Se necesita más evidencia al respecto para realizar recomendaciones razonables.

En la TVe y la TVM es necesario indagar en su causa. De forma análoga a la TP, se aconseja tratamiento anticoagulante en procesos resolubles o controlables. No existe consenso de tratamiento en las afectaciones tumorales.

Queda todavía por definir el tiempo ideal de anticoagulación en todos los supuestos. Diversos estudios aconsejan 3-6 meses en caso de factores de riesgo transitorios. En pacientes con SMP, portadores de la mutación del factor $\mathrm{V}$, del gen de la protrombina, o portadores de JAK2, debe considerarse anticoagulación indefinida. En la TP en pacientes cirróticos pendientes de trasplante, parece adecuado prolongar la anticoagulación hasta el acto quirúrgico.

Respecto a los fármacos, los mejores estudiados son HBPM, aunque en pacientes sin neoplasia puede plantearse transición hacia AVK. Sólo hay datos anecdóticos del uso de anticoagulantes de acción directa (ACOD). Pero, en caso de decidir tratamiento, este debe ser lo más precoz posible para incrementar las posibilidades de recanalización.

\section{Bibliografía}

1. Gollin G, Ward B, Meier GH, Sumpio BE, Gusberg RJ. Central splanchnic venous thrombosis. Often unsuspected, usually uncomplicated. J Clin Gastroenterol. 1994; 18:109-13.

2. Okuda K, Ohnishi K, Kimura K, Matsutani S, Sumida M, Goto N, et al. Incidence of portal vein thrombosis in liver cirrhosis. An angiographic study in 708 patients. Gastroenterology. 1985 89:279-86.

3. Cagin YF, Atayan Y, Erdogan MA, Dagtekin F, Colak C. Incidence and clinical presentation of portal vein thrombosis in cirrhotic patients. Hepatobiliary Pancreat Dis Int. 2016; 15:499-503.

4. Ahmed M, Aziz MU, Mansoor MA, Anwar S. Vascular complications in cases of acute pancreatitis - CT scan based study. J Pak Med Assoc. 2016; 66:977-89.

5. Blumberg SN, Maldonado TS. Mesenteric venous thrombosis. J Vasc Surg Venous Lymphat Disord. 2016; 4:501-7.

6. Martens P, Nevens F. Budd-Chiari syndrome. United Eur Gastroenterol J. 2015; 3:489-500

7. Fimognari FL, Violi F. Portal vein thrombosis in liver cirrhosis. Intern Emerg Med. 2008;3:213-8.

8. Gaiani S, Bolondi L, Li Bassi S, Zironi G, Siringo S, Barbara L. Prevalence of spontaneous hepatofugal portal flow in liver cirrhosis. Clinical and endoscopic correlation in 228 patients. Gastroenterology. 1991:100:160-7.

9. Cheung TK, Lai CL, Wong BC, Fung J, Yuen MF. Clinical features, biochemical parameters, and virological profiles of patients with hepatocellular carcinoma in Hong Kong. Aliment Pharmacol Ther. 2006; 24:573-583
10. Llovet JM, Bustamante J, Castells A, Vilana R, Ayuso MC, Sala M, et al. Natural history of untreated nonsurgical hepatocellular carcinoma: rationale for the design and evaluation of therapeutic trials. Hepatology 1999; 29:62-7.

11. Fama A, Rago A, Gioiosa F, Marzano C, Latagliata R, Mammì C, et al. Budd-Chiari syndrome and splanchnic vein thrombosis: masked myeloproliferative neoplasms and JAK2V617F. Clin Ter. 2010;161:169-71

12. Wang $H$, Sun G, Zhang P, Zhang J, Gui E, Zu M, et al. JAK2 V617F mutation and 46/1 haplotype in Chinese Budd-Chiari syndrome patients. J Gastroenterol Hepatol. 2014;29:208-14

13. Ren W, Qi X, Yang Z, Han G, Fan D. Prevalence and risk factors of hepatocellular carcinoma in Budd-Chiari syndrome: a systematic review. Eur J Gastroenterol Hepatol. 2013; 25:830-41.

14. Valla D, Le MG, Poynard T, Zucman N, Rueff B, Benhamou JP. Risk of hepatic vein thrombosis in relation to recent use of oral contraceptives. A case-control study. Gastroenterology. 1986; 90:807-11.

15. Khuroo MS, Datta DV. Budd-Chiari syndrome following pregnancy. Report of 16 cases, with roentgenologic, hemodynamic and histologic studies of the hepatic outflow tract. Am J Med. 1980; 68:113-21.

16. Young ID, Clark RN, Manley PN, Groll A, Simon JB. Response to steroids in BuddChiari syndrome caused by idiopathic granulomatous venulitis. Gastroenterology. 1988;94:503-7.

17. Dedania N, Agrawal N, Winter JM, Koniaris LG, Rosato EL, Sauter PK, et al. Splenic vein thrombosis is associated with an increase in pancreas-specific complications and reduced survival in patients undergoing distal pancreatectomy for pancreatic exocrine cancer. J Gastrointest Surg. 2013:17:1392-8.

18. Bernades P, Baetz A, Lévy P, Belghiti J, Menu Y, Fékété F. Splenic and portal venous obstruction in chronic pancreatitis. A prospective longitudinal study of a medicalsurgical series of 266 patients. Dig Dis Sci. 1992;37:340-6.

19. Abu-Daff S, Abu-Daff N, Al-Shahed M. Mesenteric venous thrombosis and factors associated with mortality: a statistical analysis with five-year follow-up. J Gastrointest Surg. 2009; 13:1245-50

20. Rhee RY, Gloviczki P, Mendonca CT, Petterson TM, Serry RD, Sarr MG et al. Mesenteric venous thrombosis: still a lethal disease in the 1990s. J Vasc Surg. 1994; 20:688-97.

21. El Sebay HM, Safan MA, Daoud AA, Tayel SI, Nouh MA, El Shafie S. Association of factor V Leiden, Janus kinase 2, prothrombin, and MTHFR mutations with primary BuddChiari syndrome in Egyptian patients. J Gastroenterol Hepatol. 2016; 31:235-40.

22. De Stefano V, Martinelli I. Splanchnic vein thrombosis: clinical presentation, risk factors and treatment. Intern Emerg Med. 2010; 6:487-94.

23. Darwish MS, Plessier A, Hernandez-Guerra M, Fabris F, Eapen CE, Bahr MJ, et al. Etiology, management, and outcome of the Budd-Chiari syndrome. Ann Intern Med 2009:151:167-75.

24. De Stefano V, Vannucchi AM, Ruggeri M, Cervantes F, Alvarez-Larrán A, lurlo A, et al Splanchnic vein thrombosis in myeloproliferative neoplasms: risk factors for recurrences in a cohort of 181 patients. Blood Cancer J. 2016; 6:e493.

25. Sekhar M, McVinnie K, Burroughs AK. Splanchnic vein thrombosis in myeloproliferative neoplasms. Br J Haematol. 2013; 162:730-7

26. Smalberg JH, Koehler E, Darwish Murad S, Plessier A, Seijo S, Trebicka J, et al. The JAK2 46/1 haplotype in Budd-Chiari syndrome and portal vein thrombosis. Blood. 2011; 117:3968-73.

27. Sekhar M, Patch D, Austen B, Howard J, Hart S. Calreticulin mutations and their importance in splanchnic vein thrombosis. Br J Haematol. 2016; 174:158-60.

28. Stephen E. Langabeer. CALR mutation analysis is not indicated in patients with splanchnic vein thrombosis without evidence of a myeloproliferative neoplasm: a micro-review. Ann Gastroenterol. 2016; 29: 557-8.

29. Ziakas PD, Poulou LS, Rokas Gl, Bartzoudis D, Voulgarelis M. Thrombosis in paroxysmal nocturnal hemoglobinuria: sites, risks, outcome. An overview. J Thromb Haemost 2007:5:642-5.

30. Justo D, Finn T, Atzmony L, Guy N, Steinvil A. Thrombosis associated with acute cytomegalovirus infection: a meta-analysis. Eur J Intern Med. 201; 22:195-9.

31. EASL Clinical Practice Guidelines: Vascular diseases of the liver. J Hepatol 2016; 64:179-202.

32. Seijo S, Plessier A, Hoekstra J, Dell'Era A, Mandair D, Rifai K, et al. Good longterm outcome of Budd-Chiari syndrome with a step-wise management. Hepatology 2013;57:1962-8.

33. Sharma S, Texeira A, Texeira P, Elias E, Wilde J, Olliff SP. Pharmacological thrombolysis in Budd Chiari syndrome: a single centre experience and review of the literature. J Hepatol 2004:40:172-80.

34. Tilanus HW. Budd-Chiari syndrome. Br J Surg. 1995;82:1023-30.

35. Garcia-Pagan JC, Heydtmann M, Raffa S, Plessier A, Murad S, Fabris F, et al. TIPS for Budd-Chiari syndrome: long-term results and prognostics factors in 124 patients. Gastroenterology. 2008;135:808-15.

36. Bañares R, Catalina MV. Tratamiento de la trombosis portal no tumoral en la cirrosis. Gastroenterol Hepatol. 2014;37(Supl. 2):62-7.

37. Chung JW, Kim GH, Lee JH, Ok KS, Jang ES, Jeong SH, et al. Safety, efficacy, and response predictors of anticoagulation for the treatment of nonmalignant portal-vein thrombosis in patients with cirrhosis: a propensity score matching analysis. Clin $\mathrm{Mol}$ Hepatol. 2014;20:384-91. 